\title{
Pseudo-scalar meson form factors with maximally twisted Wilson fermions at $N_{f}=2$
}

\section{S. Simula*}

Istituto Nazionale di Fisica Nucleare - Sezione di Roma Tre

Via della Vasca Navale 84, I-00146 Roma, Italy

E-mail: simula@roma3.infn.it

for the European Twisted Mass Collaboration (ETMC)

\begin{abstract}
We present preliminary results for various electroweak form factors of pseudo-scalar mesons using the tree-level improved Symanzik gauge action and the maximally twisted mass fermionic action with $N_{f}=2$ dynamical flavors. Our results, obtained for both light and heavy quark masses at a single lattice spacing ( $a \simeq 0.09 \mathrm{fm}$ ) and at a single lattice volume $\left(V * T=24^{3} * 48\right)$, exhibit a quite remarkable statistical precision thanks to the use of all-to-all quark propagators computed with a stochastic method. Moreover very low values of the four-momentum transfer are achieved by making use of twisted boundary conditions on the valence quark fields. The mass dependence of the pion charge radius is analyzed using Chiral Perturbation Theory, obtaining clear evidence of relevant two-loop contributions. The universal Isgur-Wise function is computed from heavyto-heavy electromagnetic transitions and its slope in the case of $u(d)$ spectator quarks is found to be $\rho_{I W}^{2}=0.77 \pm 0.28$, where the error is statistical only.
\end{abstract}

The XXV International Symposium on Lattice Field Theory

July 30 - August 42007

Regensburg, Germany

\footnotetext{
${ }^{*}$ Speaker.
} 


\section{Introduction}

The European Twisted Mass Collaboration (ETMC) has recently started an intensive, systematic program of calculations of three-point correlation functions using the large number of gauge configurations produced for three values of the lattice spacing and various lattice volumes adopting the tree-level improved Symanzik gauge action and the twisted mass fermionic action with $N_{f}=2$ dynamical flavors tuned at maximal twist (see [1]). The aim is the determination of the electromagnetic (e.m.) and weak semileptonic form factors relevant for light and heavy-light mesons as well as for baryons.

In this contribution we present the preliminary results obtained so far for the charge form factor of the pion, the universal Isgur-Wise (IW) function, the vector and scalar form factors relevant for $K_{\ell 3}$ decays and the $D \rightarrow K(\pi)$ transition.

The presently completed runs correspond to three simulated sea-quark masses, $a m_{\text {sea }}=0.0040$, 0.0064 and 0.0100, at $\beta=3.9$ corresponding to $a=0.087(1) \mathrm{fm}\left(a^{-1} \simeq 2.3 \mathrm{GeV}\right)$ [2], and at a single lattice volume $\left(V * T=24^{3} * 48\right)$. The mass of the spectator valence quark is fixed at the seaquark mass, while the values of the mass of the valence quark struck by the electroweak current are taken from the set $\{0.0040,0.0064,0.0085,0.0100,0.0150,0.022,0.027,0.032,0.25,0.32,0.39$, $0.46\}$. The first five masses correspond to the "light" sector and coincide with the values of the sea-quark mass adopted by the ETMC at $\beta=3.9$, the subsequent three are around the "strange" quark mass and the heaviest four lie in the range from the "charm" quark mass $m_{c}$ to twice $m_{c}$. At each value of the sea-quark mass we have computed the two- and three-point correlation functions for charged pseudo-scalar mesons, using the standard local $\gamma_{5}$ interpolating fields, on a set of 240 independent gauge configurations, separated by 20 consecutive HMC trajectories.

In order to improve the statistical accuracy we have calculated the two- and three-point correlation functions employing all-to-all quark propagators estimated through the "one-end" stochastic method of Ref. [3]. The advantages of such a procedure with respect to the "standard" one based on the use of point-to-all quark propagators with fixed point sources are clearly illustrated in Fig. 1.

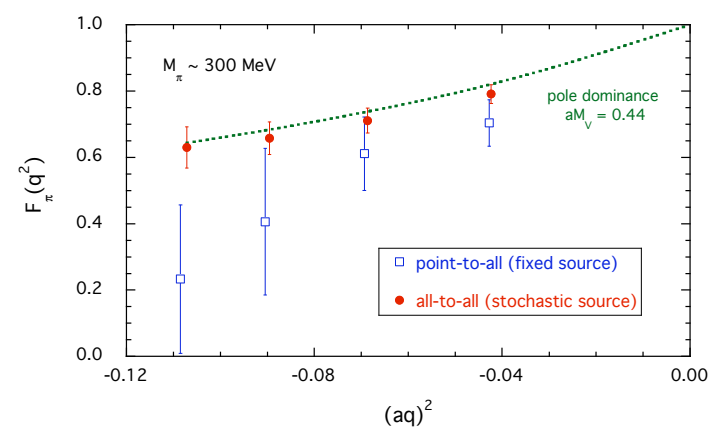

Figure 1: Charge form factor of the pion, $F_{\pi}\left(q^{2}\right)$, versus the squared four-momentum transfer $q^{2}$ in lattice units, calculated at the (bare) quark mass am $=0.0040$ using a subset of 80 ETMC gauge configurations. The dotted line is the behavior expected from vectormeson dominance at the simulated quark mass. The errors are purely statistical obtained by the jackknife procedure.

Moreover, in order to get rid of the limitations in the minimum value of the spatial momentum imposed by periodic boundary conditions, we make use of twisted boundary conditions [4] on the valence quark fields ${ }^{1}$. We have adopted the Breit frame, where initial and final mesons have opposite spatial momenta, because in such a frame for a given value of the squared four-momentum

\footnotetext{
${ }^{1}$ The use of different boundary conditions for sea and valence quarks produce finite size effects that are exponentially small [5].
} 
transfer $q^{2}$ the spatial momentum injected to the active quarks is minimized. Thus one has

$$
q^{2}=\left[\sqrt{M_{f}^{2}+\left(\frac{2 \pi}{L} \vec{\theta}\right)^{2}}-\sqrt{M_{i}^{2}+\left(\frac{2 \pi}{L} \vec{\theta}\right)^{2}}\right]^{2}-\left(\frac{2 \pi}{L} 2 \vec{\theta}\right)^{2}
$$

where $M_{i}\left(M_{f}\right)$ is the initial (final) meson mass and $\vec{\theta}$ is a real variable. In our simulations we have chosen $\vec{\theta}=(\tilde{\theta}, \tilde{\theta}, \tilde{\theta})$ with $\tilde{\theta}$ ranging from 0.1 to 1 .

Thanks to the tuning at maximal twist many physical observables at zero momentum are automatically $O(a)$-improved [6]. As for the matrix elements of the electroweak current at nonvanishing momenta, the improvement can be realized by a suitable averaging of matrix elements with meson momenta of equal magnitude but opposite sign [6]. In the Breit frame this is equivalent to the exchange of initial and final quark masses ${ }^{2}$. As a byproduct, the matrix elements of the e.m. current are automatically $O(a)$-improved at any momenta in the Breit frame.

We employ on the lattice the local vector current which needs to be renormalized. The renormalization constant $Z_{V}$ can be calculated using the matrix element of the time component of the (local) vector current between pions at rest. Indeed, since the charge form factor of the pion is normalized to unity at $q^{2}=0$, one has

$$
Z_{V}\left\langle\pi^{+}(\overrightarrow{0})\left|\frac{2}{3} \bar{u} \gamma^{0} u-\frac{1}{3} \bar{d} \gamma^{0} d\right| \pi^{+}(\overrightarrow{0})\right\rangle=2 M_{\pi}
$$

Another way to obtain $Z_{V}$ is the use of the axial Ward Identity as carried out in Ref. [8]. The two determinations exhibit a quite remarkable statistical precision $(\simeq 0.03 \%)$, and they agree very well in the chiral limit, as shown in Fig. 2, while at non-vanishing quark masses they differ mainly by terms of the order of $a^{2} m \Lambda_{Q C D}$.

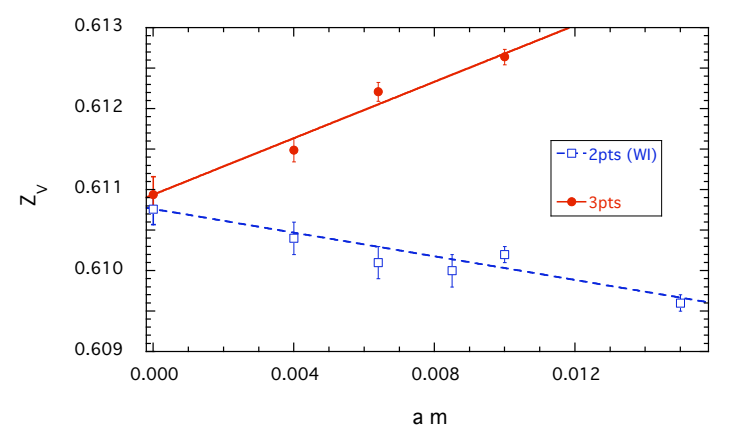

Figure 2: Renormalization constant of the local vector current $Z_{V}$ calculated via Eq. (1.2) (full dots) and through the axial Ward identity (squares) [8] for three values of the (bare) quark mass am. The errors are purely statistical obtained by the jackknife procedure.

\section{Charge form factor of the pion}

The charge form factor of the pion, $F_{\pi}\left(q^{2}\right)$, is directly related to the matrix element of the time component of the renormalized (local) e.m. current by

$$
F_{\pi}\left(q^{2}\right)=\frac{Z_{V}}{2 E_{\pi}}\left\langle\pi^{+}(\vec{\theta})\left|\frac{2}{3} \bar{u} \gamma^{0} u-\frac{1}{3} \bar{d} \gamma^{0} d\right| \pi^{+}(-\vec{\theta})\right\rangle .
$$

\footnotetext{
${ }^{2}$ Note that the added correlator is almost costless if the multisolver algorithm [7] is adopted for the inversion of the Dirac equation.
} 
where $E_{\pi}=\sqrt{M_{\pi}^{2}+(2 \pi \vec{\theta} / L)^{2}}$ and $q^{2}=-4(2 \pi \vec{\theta} / L)^{2}$. Note that the value of $q^{2}$ is independent of the simulated pion mass. The matrix element appearing in Eq. (2.1) can be extracted from a suitable ratio of three-point to two-point correlation functions. The quality of the plateaux is illustrated in Fig. 3. We remind that in the two-point correlator the identification of the pion ground state starts already at a time around $t / a=10$ (see [9]).
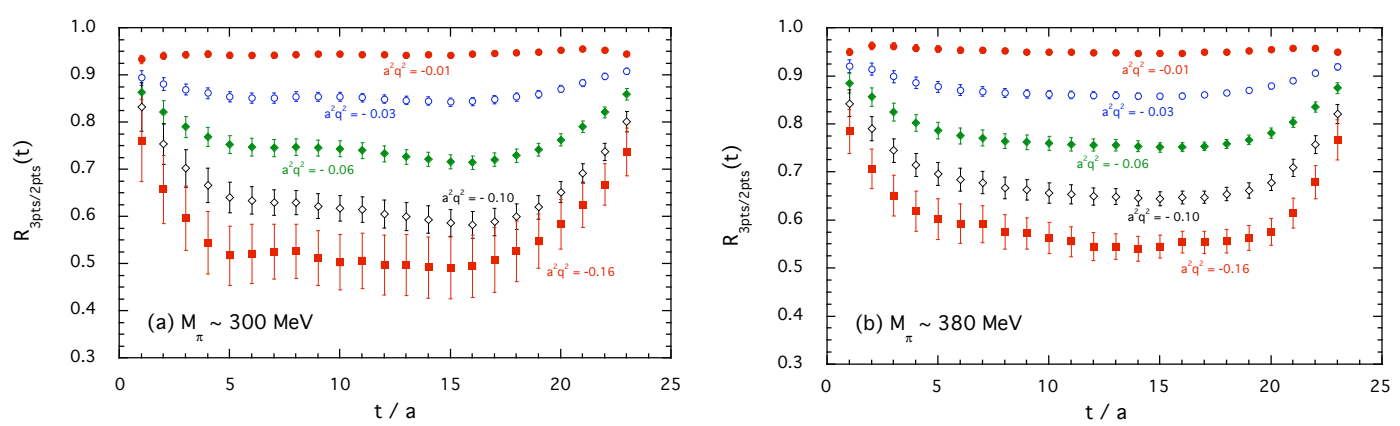

Figure 3: Ratio of three-point to two-point correlation functions, $R_{3 p t s / 2 p t s}(t)$, versus the lattice time for two values of the (bare) quark mass $a m=0.0040$ (a) and 0.0060 (b), corresponding to physical pion mass around 300 and $380 \mathrm{MeV}$, respectively. The plateaux of $R_{3 p t s / 2 p t s}(t)$ provide directly the form factor (2.1).

The results obtained for $F_{\pi}\left(Q^{2} \equiv-q^{2}\right)$ in the unitary setup (i.e., equal valence and sea quark masses) are reported in Fig. 4(a) and compared with experimental data from Ref. [10]. It can be seen that: i) the lattice results exhibit a remarkable statistical precision; ii) thanks to the use of twisted boundary conditions the form factor is precisely determined at values of $Q^{2}$ as low as $0.05 \mathrm{GeV}^{2}$; and iii) the lattice results, obtained at pion masses of about 300,380 and $470 \mathrm{MeV}$, overestimate the experimental data in the whole range of values of $Q^{2}$.
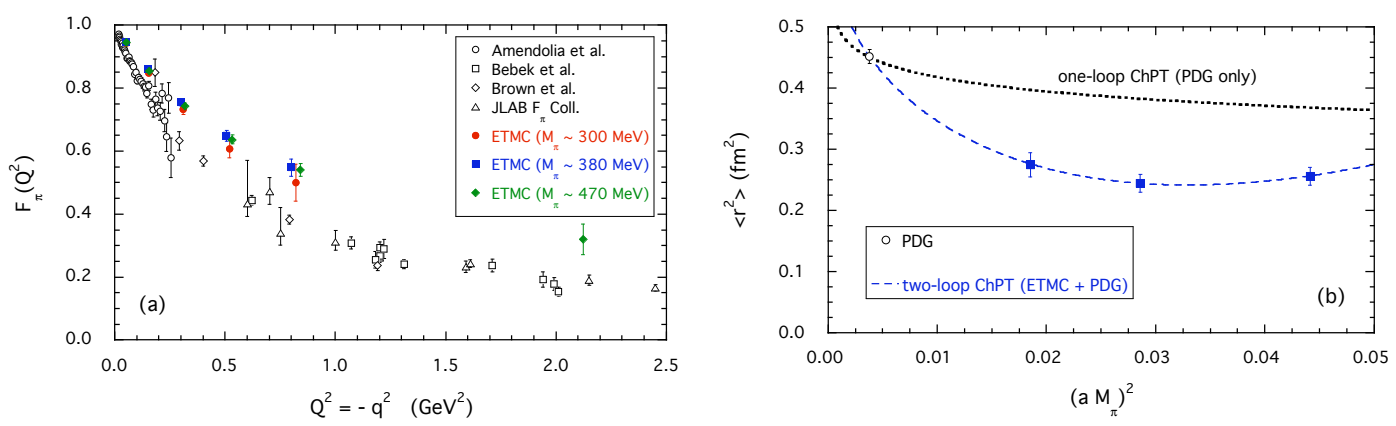

Figure 4: (a) Charge form factor of the pion $F_{\pi}\left(Q^{2}\right)$ versus $Q^{2} \equiv-q^{2}$ (in physical units). The open markers are the experimental data of Ref. [10], while the full markers are the ETMC results. The lattice points at $M_{\pi} \sim 380$ and $470 \mathrm{MeV}$ are slightly shifted in $Q^{2}$ for a better reading. (b) Squared pion charge radius (in physical units) versus the squared pion mass (in lattice units). The experimental point is from PDG [11]. The dotted and dashed lines are the results of ChPT fits at one-loop and two-loops, respectively (see text).

The $q^{2}$-dependence of our ETMC results can be very nicely fitted assuming a pole behavior. The corresponding values of the pion charge radius are shown in Fig. 4(b) and lie well below the experimental value from PDG [11].

The predictions of Chiral Perturbation Theory (ChPT) for the pion charge radius have been elaborated at one-loop in Ref. [12] and at two-loop in Ref. [13] (in the continuum and infinite 
volume limits). At one-loop one has

$$
\left\langle r^{2}\right\rangle=\frac{2}{(4 \pi F)^{2}}\left[\log \left(\Lambda_{6}^{2} / M_{\pi}^{2}\right)-1\right],
$$

where $F$ is the pion decay constant in the chiral limit and $\Lambda_{6}$ a low-energy constant (LEC), while the two-loop formula of Ref. [13] can be rewritten as

$$
\left\langle r^{2}\right\rangle=\frac{2}{(4 \pi F)^{2}}\left[\log \left(\Lambda_{6}^{2} / M_{\pi}^{2}\right)-1\right]+A M_{\pi}^{2}+B M_{\pi}^{2} \log \left(M_{\pi}^{2}\right),
$$

where $A$ and $B$ depend on various LEC's. The value of the decay constant $F$ has been determined for our unitary setup in Ref. [2] ( $a F=0.0534(6)$ corresponding to $F \simeq 121 \mathrm{MeV}$ ). Thus the experimental value of the pion charge radius fix the value of the LEC $\Lambda_{6}$ in the one-loop formula (2.2), namely $\overline{\ell_{6}} \equiv\left[\log \left(\Lambda_{6}^{2} / M_{\pi}^{2}\right)\right]_{M_{\pi}=139.6 \mathrm{MeV}}=14.4(3)$, as well as its mass dependence, as shown in Fig. 4(b) by the dotted line. The differences of the one-loop ChPT prediction and the ETMC points represent a clear indication of important contributions from higher loops.

In this preliminary analysis we neglect both finite size and discretization effects, which are nevertheless expected to be small, and we use the two-loop formula (2.3), containing three free parameters, to fit both the three ETMC points and the PDG value, obtaining $\overline{\ell_{6}}=17.2(7)$, as shown by the dashed line in Fig. 4(b)). It can be seen that the chiral enhancement expected at low pion masses is hardly visible at the simulated pion masses. More lattice points, particularly below $M_{\pi} \sim 300 \mathrm{MeV}$, are necessary to find out a clear signature of the chiral logs.

We have calculated also the scalar form factor of the pion, limiting ourselves only to the connected insertion of the scalar density operator. The results will be presented elsewhere [14]. We simply want to point out that the values obtained for the scalar radius exhibit the same qualitative features of those discussed above for the pion charge radius, including the relevance of two-loop effects in their pion mass dependence.

\section{Universal Isgur-Wise function}

The investigation of heavy-to-heavy e.m. transitions, described by a single form factor

$$
F_{P S}\left(q^{2}\right)=\frac{Z_{V}}{2 E_{P S}}\left\langle P S(\vec{\theta})\left|\bar{h} \gamma^{0} h\right| P S(-\vec{\theta})\right\rangle,
$$

allows to determine the IW function $\xi(\omega)$ by performing the infinite heavy-quark limit, viz. $\xi(\omega)=$ $\lim _{m_{h} \rightarrow \infty} F_{P S}\left(q^{2}\right)$, where $\omega=1-q^{2} / 2 M_{P S}^{2}{ }^{3}$. Note that the form factor $F_{P S}\left(q^{2}\right)$ is automatically normalized to unity at $q^{2}=0$ for any simulated mass because of the conservation of the e.m. current.

We have calculated $F_{P S}\left(q^{2}\right)$ for various values of the (bare) heavy-quark mass, am ${ }_{h}$, taking the spectator-quark mass, $a m_{s p}$, to be equal to the sea-quark mass $a m_{s e a}$. In Fig. 5(a) we have reported the results obtained at the lowest sea-quark mass, $a m_{s p}=a m_{\text {sea }}=0.0040$. It can be seen that the dependence upon the heavy-quark mass is very mild so that the extrapolation to the infinite heavy-quark limit can be safely neglected.

\footnotetext{
${ }^{3}$ With such a definition we disregard the (small) perturbative correction that should be removed from Eq. (3.1) to get the proper renormalization-group invariant definition of $\xi(\omega)$.
} 

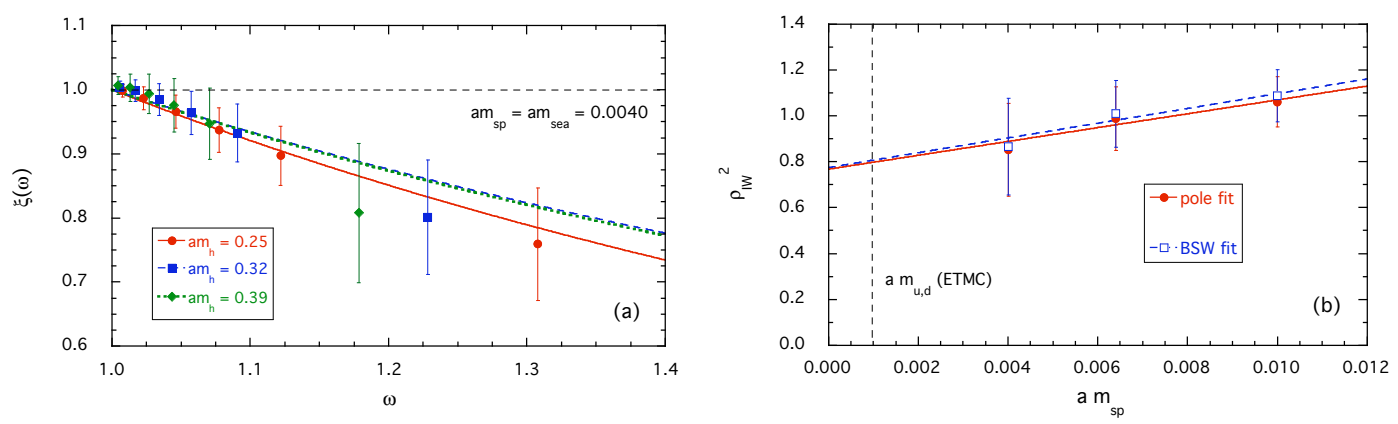

Figure 5: (a) The IW function calculated at $a m_{s p}=a m_{\text {sea }}=0.0040$ for various values of the heavy-quark mass $a m_{h}$. The various lines are fits based on a "pole" ansätz. (b) Values of the IW slope $\rho_{I W}^{2}$ obtained through "pole" (full dots) and BSW (open squares) ansätz. The vertical line correspond to the value of the (bare) light $u(d)$ quark mass determined in lattice units in Ref. [9].

Our results for $\xi(\omega)$ can be nicely fitted using either the pole or the BSW [15] ansätz. The corresponding values of the IW slope $\rho_{I W}^{2} \equiv-[d \xi(\omega) / d \omega]_{\omega=1}$ are reported in Fig. 5(b). A naïve linear extrapolation in the spectator quark mass to the (bare) light $u(d)$ quark mass, as determined by ETMC in Ref. [9], gives $\rho_{I W}^{2}=0.77 \pm 0.28$, where the error is statistical only. To our knowledge this is the first determination of the IW slope with $N_{f}=2$. Recently a quite precise value of the IW slope, $\rho_{I W}^{2}=0.89 \pm 0.17$, has been obtained ain the quenched approximation in Ref. [16].

\section{4. $K_{\ell 3}$ decays and heavy-to-light transitions}

As it is known, the matrix element of the vector weak current between pseudo-scalar mesons involve two form factors, the vector $f_{+}\left(q^{2}\right)$ and the scalar $f_{0}\left(q^{2}\right)$ ones, namely:

$$
\begin{aligned}
\left\langle P S_{2}\left|V_{\mu}^{(\text {weak })}\right| P S_{1}\right\rangle & =\left(p_{1}+p_{2}\right)_{\mu} f_{+}\left(q^{2}\right)+\left(p_{1}-p_{2}\right)_{\mu} f_{-}\left(q^{2}\right) \\
f_{0}\left(q^{2}\right) & \equiv f_{+}\left(q^{2}\right)+\frac{q^{2}}{M_{1}^{2}-M_{2}^{2}} f_{-}\left(q^{2}\right)
\end{aligned}
$$

where $q^{2}=\left(p_{1}-p_{2}\right)^{2}$. In this contribution we limit ourselves to illustrate in Fig. 6 the nice level of statistical precision achieved in the determination of the vector and scalar form factors relevant for the cases of $K_{\ell 3}$ decays and of the $D \rightarrow K$ transition.
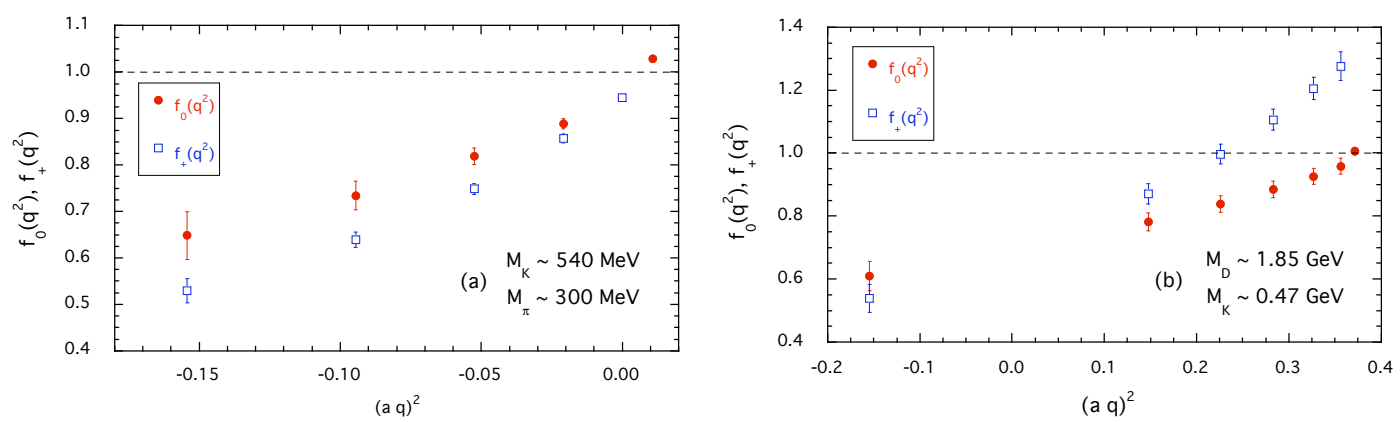

Figure 6: Vector $f_{+}\left(q^{2}\right)$ and scalar $f_{0}\left(q^{2}\right)$ form factors of pseudo-scalar mesons relevant for $K_{\ell 3}$ decays (a) and for the $D \rightarrow K$ transition (b). 


\section{Conclusions}

We have presented preliminary results for the electroweak form factors of light and heavy-light pseudo-scalar mesons, obtained at a single lattice spacing $(a \simeq 0.09 \mathrm{fm})$ and at a single lattice volume $\left(V * T=24^{3} * 48\right)$, using the tree-level improved Symanzik gauge action and the twisted mass Wilson action with $N_{f}=2$ dynamical flavors tuned at maximal twist. The use of all-to-all quark propagators computed with a stochastic method, as well as of twisted boundary conditions on the valence quark fields has allowed us to achieve both a quite remarkable statical precision and very low values of the four-momentum transfer. We have analyzed the mass dependence of the pion charge radius using Chiral Perturbation Theory, obtaining evidence of relevant two-loop contributions. Results at more values of the sea quark mass, as well as the investigation of volume effects and continuum extrapolation, are however required in order to draw definite quantitative conclusions. The universal Isgur-Wise function has been computed from heavy-to-heavy electromagnetic transitions and its slope in the case of $u(d)$ spectator quarks has been found to be $\rho_{I W}^{2}=0.77 \pm 0.28$, where the error is statistical only.

\section{References}

[1] ETM Collaboration, C. Urbach (plenary talk), PoS(LATTICE 2007)022 arXiv:0710.1517 [hep-lat].

[2] Ph. Boucaud et al. [ETM Collaboration], Phys. Lett. B 650 (2007) 304 [arXiv:hep-lat/0701012].

[3] M. Foster, C. Michael [UKQCD Coll.], Phys. Rev. D 59 (1999) 074503 [arXiv:hep-lat/9810021]. C. McNeile, C. Michael [UKQCD Coll.], Phys. Rev. D 73 (2006) 074506 [arXiv:hep-lat/0603007].

[4] P. F. Bedaque, Phys. Lett. B 593 (2004) 82 [arXiv:nucl-th/0402051]. G. M. de Divitiis, R. Petronzio and N. Tantalo, Phys. Lett. B 595 (2004) 408 [arXiv:hep-lat/0405002]. D. Guadagnoli, F. Mescia and S. Simula, Phys. Rev. D 73 (2006) 114504 [arXiv:hep-lat/0512020].

[5] C. T. Sachrajda and G. Villadoro, Phys. Lett. B 609 (2005) 73 [arXiv:hep-lat/0411033].

[6] R. Frezzotti and G. C. Rossi, JHEP 0408 (2004) 007 [arXiv:hep-lat/0306014].

[7] B. Jegerlehner, Nucl. Phys. Proc. Suppl. 63 (1998) 958 [arXiv:hep-lat/9708029].

[8] ETM Collaboration, P. Dimopoulos et al., PoS(LATTICE 2007)241 arXiv:0710.0975 [hep-lat].

[9] ETM Collaboration, V. Lubicz et al., PoS(LATTICE 2007)374 arXiv:0710.0329 [hep-lat]. B. Blossier et al., arXiv:0709.4574 [hep-lat].

[10] S.R. Amendolia et al., Nucl. Phys. B277 (1986) 168; Phys. Lett. B 146 (1984) 116. C.J. Bebek et al., Phys. Rev. D 9 (1974) 1229; Phys. Rev. D 13 (1976) 25; Phys. Rev. D 17 (1978) 1693. C.N. Brown et al., Phys. Rev. D 8 (1973) 92. V. Tadevosyan et al. [JLab F(pi) Collaboration], Phys. Rev. C 75 (2007) 055205 [arXiv:nucl-ex/0607007]. T. Horn et al. [JLab F(pi)-2 Collaboration], Phys. Rev. Lett. 97 (2006) 192001 [arXiv:nucl-ex/0607005]; T. Horn et al., arXiv:0707.1794 [nucl-ex].

[11] W.M. Yao et al. [Particle Data Group Coll.], J. Phys. G 33 (2006) 1.

[12] J. Gasser and H. Leutwyler, Annals Phys. 158 (1984) 142.

[13] J. Bijnens, G. Colangelo and P. Talavera, JHEP 9805 (1998) 014 [arXiv:hep-ph/9805389].

[14] S. Simula et al. [ETM Collaboration], in preparation.

[15] M. Wirbel, B. Stech and M. Bauer, Z. Phys. C 29 (1985) 637.

[16] G. M. de Divitiis, R. Petronzio and N. Tantalo, arXiv:0707.0587 [hep-lat]. 